\title{
Cinéma et genre en France : état des lieux
}

\section{Geneviève SELLIER et Brigitte ROLLET}

\section{(2) OpenEdition}

\section{Journals}

Édition électronique

URL : https://journals.openedition.org/clio/1533

DOI : 10.4000/clio.1533

ISSN : 1777-5299

Éditeur

Belin

\section{Édition imprimée}

Date de publication : 1 novembre 1999

ISBN : 2-85816-483-5

ISSN : $1252-7017$

\section{Référence électronique}

Geneviève SELLIER et Brigitte ROLLET, «Cinéma et genre en France : état des lieux », Clio. Histoire,

femmes et sociétés [En ligne], 10 | 1999, mis en ligne le 22 mai 2006, consulté le 23 avril 2022. URL http://journals.openedition.org/clio/1533 ; DOl : https://doi.org/10.4000/clio.1533

Ce document a été généré automatiquement le 23 avril 2022.

Tous droits réservés 


\title{
Cinéma et genre en France : état des lieux
}

\author{
Geneviève SELLIER et Brigitte ROLLET
}

\section{NOTE DE L'ÉDITEUR}

Brigitte Rollet tient à remercier la British Academy pour son soutien lors des recherches nécessaires à la rédaction de cet article.

1 Parler d'« exception culturelle » française est parfois pleinement justifié. Ainsi, dès lors que sont étudiés de près les rapports pour le moins conflictuels entre genre et cinéma en France, certaines tendances spécifiques se dessinent. Il n'est pas nouveau de dire que l'équivalent français du terme anglais gender a quelques difficultés à s'intégrer dans le monde universitaire hexagonal et les réticences à envisager la société au sens large en fonction des sexes biologiques et des rapports entre les sexes se retrouvent dans tous les domaines de recherche. Après les revendications des femmes dans les années 1970 qui plaçaient leur appartenance de sexe au cœur des débats et des luttes, la tendance semble être revenue à une neutralisation des genres, et ce malgré les récents combats pour la parité et la féminisation des noms de métiers. Parler par exemple de la nécessité ou même de l'opportunité d'un festival de films de femmes semble relever de la ringardisation absolue.

Dans le monde cinématographique français en effet, les paradoxes sont à la fois nombreux et surprenants (surtout pour les universitaires étranger-e-s). Dans un pays où les femmes représentent, depuis les années 1970, entre 15 et $20 \%$ de l'ensemble des cinéastes, ce qui est une situation unique au monde, ces mêmes femmes sont parfois les premières à refuser que l'on considère leurs œuvres comme "genrées ». Ces refus se “justifient" lorsque l'on considère une autre donnée majeure et tout aussi paradoxale. Quoiqu'ayant "exporté" ses théories outre-Manche et outre-Atlantique, le monde intellectuel et universitaire français n'a jamais été très enclin à importer ce à quoi ces mêmes théories ont donné lieu depuis plus d'un quart de siècle dans les pays anglo- 
saxons. Ainsi, les travaux de Pierre Bourdieu ont-ils été intégrés avec succès au développement des Cultural studies originaires de Birmingham (Grande-Bretagne) et représentent aujourd'hui la pierre angulaire d'une approche privilégiant les conditions de production (et de reproduction) des pratiques et productions culturelles. L'écart entre la France et les pays anglo-saxons est donc frappant dans le champ des études filmiques et des études culturelles en général. La constitution de ces champs de recherche dans les années 1960 et 1970 en Grande-Bretagne et aux États-Unis s'est faite dans un contexte politique de contestation du savoir académique, à partir d'une critique du pouvoir patriarcal et de l'élitisme culturel qui ne connaît pas d'écho en France, même si, paradoxalement, ce sont en partie des textes de théoricien-ne-s français-es qui ont été utilisés comme soubassement à l'émergence de ces nouvelles disciplines ${ }^{1}$.

En ce qui concerne les publications inspirées de théories non françaises (même si le cadre méthodologique l'est), l'écart est encore plus flagrant. Cette situation est à rapprocher de la situation assez particulière de l'enseignement supérieur en France où le cloisonnement des disciplines est encouragé, pour ne pas dire imposé, et où la notion d'interdisciplinarité est peu, et le plus souvent pas envisagée ${ }^{2}$. Pour le cinéma donc, l'approche prédominante reste la tendance formaliste ("exportée" elle aussi, mais dont les chercheurs anglo-saxons ont su se défaire plus rapidement qu'en France) et esthétique. L'idée d'envisager l'œuvre dans le contexte plus large de la société qui l'a produite, en tenant compte de critères tels que le sexe, l'âge, la classe sociale ou l'appartenance "ethnique", sans oublier les conditions particulières de production et de distribution, ainsi que les liaisons parfois dangereuses entre la culture en France et le pouvoir politique, reste ultra minoritaire, pour ne pas dire ignorée ${ }^{3}$. Tout comme le structuralisme négligeait «tout ce qui excéd[ait] la sphère du discours »" le film est souvent enseigné indépendamment des conditions d'émission, de production et de réception qui en structurent le sens.

4 Pour ce qui est des sexes et des rapports de sexe dans le cinéma français, le champ d'investigation et de recherche est pourtant vaste. Outre l'analyse des rapports sexués dans les productions filmiques et donc des représentations où prédomine un regard traditionnellement et majoritairement masculin, l'étude de la place et du rôle des femmes au sein de l'industrie cinématographique ouvre des horizons encore peu défrichés en France. Auteures d'un cinéma souvent qualifié de "mineur" (et plus particulièrement dans les années 1970), les réalisatrices françaises sont souvent cantonnées aux genres du même nom ${ }^{5}$.

5 En ce qui concerne les théories féministes sur le cinéma, que Ginette Vincendeau et Béatrice Reynaud ont tenté de faire connaître au public français en traduisant des extraits des textes fondateurs ${ }^{6}$, la situation est encore plus surprenante. Les gender studies qui s'intéressent aux identités et aux rapports de sexes en tant que constructions socioculturelles, ne trouvent pas d'équivalent en français, le mot "sexe" étant utilisé pour traduire aussi bien le sexe biologique que le sexe social. Alors que les cultural studies se sont d'abord intéressées à d'autres disciplines que le cinéma, les gender studies ont trouvé très tôt un terrain de recherche privilégié dans l'étude des films ${ }^{7}$. L'article de Laura Mulvey "Visual Pleasure and Narrative Cinéma», publié en 1975 dans la revue britannique $S_{c r e e n}{ }^{8}$, représente un moment clé de la théorisation des gender studies sur le cinéma. Ce texte ouvrait la réflexion à une critique du cinéma hollywoodien comme instrument de la domination patriarcale, à travers l'analyse 
sémiologique et psychanalytique des codes du montage narratif classique. Reprenant les concepts freudiens de fétichisme, de voyeurisme et de scopophilie, Mulvey analysait le cinéma classique hollywoodien comme construit sur trois types de regards : celui de la caméra, fondamentalement voyeuriste et traditionnellement et majoritairement masculin, puisque contrôlé par un cinéaste homme; le regard des personnages masculins construit sur le système des champs-contrechamps qui assignent aux personnages féminins la position d'objet; enfin, le regard du spectateur, forcé de s'identifier aux deux premiers. Ce texte a mené à de multiples élaborations théoriques et critiques, permettant d'élargir et d'affiner à la fois la démarche liée au genre offerte par le cinéma hollywoodien, principalement à partir de la psychanalyse freudienne et lacanienne. L'ensemble de ces études visent à définir et à comprendre la position de spectatrice dans le texte filmique et à analyser les enjeux théoriques et critiques de la création filmique au féminin'. En outre, dans une perspective sémio-socioculturelle, des recherches se sont multipliées sur la dimension sexuée des grands genres populaires ${ }^{10}$, sur une approche renouvelée des grands cinéastes classiques ${ }^{11}$, sur la spécificité du public féminin et du regard des femmes sur les films, enfin sur les trop rares et sousestimées cinéastes femmes ${ }^{12}$.

6 Ce nouveau regard sexué sur le cinéma a conduit des chercheurs-ses à s'interroger sur la construction de la masculinité dans les représentations filmiques, démarche brillamment illustrée par Ginette Vincendeau dans son étude sur Gabin (voir plus loin). La prise en compte de l'identité sexuée du public a permis des investigations nouvelles sur d'autres dimensions de l'identité sociale utilisées par les cultural studies telles la classe, l'appartenance "ethnique", la religion, etc. ${ }^{13}$. Liés au mouvement de reconnaissance des "minorités sexuelles" en cours depuis une dizaine d'années dans les pays anglo-saxons, de nombreux travaux étudient aussi les textes gays et lesbiens qui sous-tendent certains films classiques ${ }^{14}$, la réception des films en fonction de l'orientation sexuelle des spectateurs-trices ${ }^{15}$, ou encore les œuvres produites par des cinéastes homosexuel-le-s ${ }^{16}$. Plus récemment encore, les queer studies vont plus loin, remettant en cause l'idée même de l'assignation à une identité de sexe, optant pour un "nomadisme" identitaire et culturel ${ }^{17}$.

7 Nous avons souhaité dans cette introduction présenter un état des recherches sur le sujet, tenant compte du sous-développement de ces approches en France. Ceci nous a conduit à ne mentionner que les ouvrages majeurs dans ce domaine, le nombre de publications en anglais allant croissant depuis plus de vingt ans. Avec l'ouvrage de Ginette Vincendeau et de Béatrice Reynaud (1993) déjà cité, le livre de Noël Burch, Revoir Hollywood (1994), est l'un des rares à offrir des textes fondateurs traduits. Afin d'illustrer l'apport indéniable des gender studies et des cultural studies, nous avons choisi de présenter de façon plus approfondie quelques ouvrages permettant d'appréhender les thèmes majeurs de l'analyse filmique dans une perspective genrée. Certains de ces thèmes font partie de l'approche traditionnelle du cinéma mais ils sont traités ici d'un point de vue féministe : il s'agit des genres cinématographiques et des auteurs. D'autres sont plus spécifiques aux "études culturelles" et s'attachent pour l'un à la réception des films pour un public féminin précis, et pour l'autre au statut particulier de la star et son rôle dans la création du sens, ou pour reprendre les termes de Richard Dyer, l'un des spécialistes britanniques des stars studies ${ }^{18}$, la star comme signe.

Dans l'étude magistrale de Ginette Vincendeau sur Jean Gabin ${ }^{19}$, il s'agit tout d'abord du mythe, puis de la masculinité et enfin de la francité. Cet ouvrage allie de façon 
extrêmement convaincante les études culturelles à l'anglo-saxonne et les théories sur le gender au cinéma. L'étude de Gabin en tant que star est ce qui intéresse tout d'abord Vincendeau. À travers une analyse du personnage mythique que l'acteur est devenu au fil de sa carrière, l'auteure s'interroge sur le pourquoi et le comment de ce mythe. À partir de l'analyse de la star comme "pure construction médiatique" et "personne réelle", et comme "circulation triangulaire" entre l'homme, l'acteur et le personnage, Vincendeau démontre le fonctionnement du mythe dans les films de Gabin. Cette analyse méticuleuse lui permet de mettre en évidence ensuite un autre aspect du mythe : le rôle de l'acteur dans la perception et la construction d'une identité nationale spécifique. Français, Gabin l'est selon Vincendeau dans toutes les représentations filmiques qu'il offre à travers sa carrière cinématographique, avec toutes les ambiguïtés que cela suppose d'une décennie à l'autre. Des personnages de prolétaires de ses débuts à ceux de grands bourgeois de la fin de sa carrière, du Front Populaire au poujadisme, l'acteur Gabin a traduit sur l'écran sa propre ascension sociale tout en gardant les signes associés au "populaire". À partir de ce que Bourdieu décrit dans La Distinction (1979) comme entrant dans la formation d'"un corps de classe", Vincendeau analyse ce qui fait de Gabin un héros "prolétarien". L'étude du physique, du maintien, du langage, ou encore de la nourriture des personnages qu'il interprète, permet de situer Gabin dans une tradition culturelle valorisant l'homme d'origine modeste par rapport au bourgeois. Définie socialement par des pratiques liées à sa "classe", la personnalité fictive de Gabin est aussi envisagée par rapport à l'“autre", ici à ceux et celles originaires d'autres cultures et/ou d'autres continents. La dernière partie de l'étude consacrée à "Gabin homme" permet à l'auteure d'analyser la construction de la masculinité dans les films et les personnages de Gabin, montrant comment la star dépasse la situation classique de construction des "regards" telle que l'a définissait Mulvey, pour devenir lui-même l'objet (mais aussi sujet) du regard.

9 Kathleen Rowe (1995) et Lucy Fischer (1996) s'intéressent plus particulièrement aux genres cinématographiques : la première se consacre exclusivement à la comédie et aux genres comiques, la seconde part d'un thème majeur dans "l'histoire des femmes", la maternité, qu'elle traite à travers divers genres cinématographiques, offrant pour chacun une démarche à la fois diachronique et synchronique .

L'originalité du travail de Rowe tient aux multiples perspectives qu'elle offre sur un genre, qui, quoique extrêmement populaire depuis les débuts du septième art, a rarement été analysé en profondeur. Ici, elle étudie les rapports souvent difficiles entre les femmes et les genres comiques, aussi bien au niveau de la création que de la réception. Cette ambivalence, ou plus exactement ce décalage qui existe entre l'identité sexuelle des rieuses et l'identification avec le personnage comique (jusqu'à récemment de sexe masculin) est envisagé par l'auteure comme la conséquence du fonctionnement même de la plaisanterie. À partir de l'étude de Freud sur Les Mots d'esprits et leur rapport avec l'inconscient, Rowe montre que l'exclusion des femmes est due au mécanisme de la plaisanterie, cette dernière posant systématiquement les femmes en situation de victimes. Selon Rowe, trois personnages sont nécessaires pour que la plaisanterie fonctionne: la personne qui la fait, celle qui en rit et celle dont on rit, l'humour s'exerçant par définition aux dépens de quelqu'un(e). L'ouvrage de Freud distribue les rôles comme suit: les deux premiers sont tenus par des hommes, et celui de la victime par une femme. Cette démonstration permet à l'auteure d'aller plus loin en prouvant combien la comédie, genre masculin par excellence tant au niveau de la réalisation que des acteurs, fut pendant des décennies peu favorable aux femmes, étant de plus par 
nature, conservatrice et rétrograde. Rejoignant les travaux de Mary Ann Doane sur le public féminin (1982 et 1988-89), elle analyse le positionnement des femmes spectatrices par rapport à la comédie, partagées entre l'identification "masochiste" avec les "objets" de la plaisanterie, en d'autres termes les personnages féminins maltraités, ou l'identification "travestie" avec les "sujets", i.e.: les protagonistes masculins à l'origine du rire. L'auteure montre l'évolution de ce genre après mai 1968 et les mouvements féministes. Elle suit l'émergence du comique au féminin à travers la réappropriation progressive du rire par les femmes, cinéastes et comédiennes, devenant à leur tour "sujet" du rire et non plus cible des rieurs-ses. Elle définit les diverses tendances de la femme "sans règle" (unruly) dont le comportement est fait de transgressions variées, qu'elles soient linguistiques, physiques, etc. Bien que son étude porte principalement sur le cinéma originaire des États-Unis, son analyse et sa méthodologie sont applicables à d'autres cinémas nationaux.

11 Il en est de même pour l'ouvrage de Lucy Fischer. Celle-ci reprend à son compte des études précédentes sur les relations entre cinéma et figure/s maternelle/s auxquelles elle consacre son chapitre introductif. Comme les autres livres dont il est question ici, elle utilise l'apport des cultural studies et des gender studies, montrant, s'il en était besoin, les liens étroits entre les deux disciplines et théories. Partant de l'image maternelle récurrente dans le cinéma depuis les origines du septième art, Fischer analyse les multiples variations offertes par les différents genres cinématographiques (fiction et documentaire) depuis les films muets jusqu'à nos jours, tendant dans leur ensemble à présenter la mère comme étant à la fois le symbole et la métaphore des crises. Donnée incontournable des mouvements de femmes, qu'elle soit niée ou revendiquée, la maternité est envisagé ici dans une perspective assez large, l'auteure utilisant de nombreux outils méthodologiques (psychanalyse, anthropologie, histoire et sociologie) pour relever les tendances inhérentes à chaque genre dans le traitement de la maternité.

12 Sandy Flitterman-Lewis (1996) propose d'articuler approche historique, sémiologique et psychanalytique pour examiner l'œuvre de trois cinéastes françaises qui participèrent à trois moments forts de la construction du cinéma français comme une alternative au modèle hollywoodien dominant: Germaine Dulac dans les années 1920, Marie Epstein dans les années 1930, Agnès Varda dans les années 1960.

13 Flitterman réévalue la carrière et l'œuvre de ces trois cinéastes, situe l'œuvre dans son contexte historique, économique et esthétique, et explore les différentes façons qu'ont ces œuvres de construire un regard féminin et de remettre en cause les représentations patriarcales de l'expérience féminine.

14 Germaine Dulac, théoricienne et cinéaste de la "première avant-garde" des années 1920, avec Delluc, Epstein, Gance et L'Herbier, décrit dans La Souriante Madame Beudet (1922) l'existence d'une femme au foyer de milieu bourgeois qui cherche à se libérer en laissant libre cours à son imagination. L'intrigue sert de prétexte pour explorer les méandres d'une subjectivité féminine, dont les fantasmes sont figurés sur le mode parodique et poétique.

15 Marie Epstein, sœur et collaboratrice du théoricien-cinéaste Jean Epstein (comme scénariste notamment) a réalisé avec Jean-Benoît Lévy (qui en est le seul crédité dans les dictionnaires et histoires du cinéma...) trois films muets et huit films parlants avantguerre. Flitterman examine le plus connu d'entre eux, La Maternelle (1934), avec Madeleine Renaud, d'après le roman de Léon Frappié. L'adaptation se focalise sur la 
petite Marie, abandonnée par sa mère, une prostituée, et qui reporte son amour sur Rose, l'aide-maternelle de l'école. Quand la petite fille se sent menacée par le projet de mariage de la jeune femme, elle tente de se suicider. La réconciliation finale est permise par l'acceptation par Marie du mariage de Rose. Flitterman montre que l'originalité du film est de construire le processus d'identification du spectateur à partir du point de vue d'une petite fille et de son amour pour une figure de mère. Elle analyse en détail les séquences qui mettent en scène de façon récurrente le traumatisme de la scène primitive d'un point de vue féminin, prenant à contre-pied la trajectoire œdipienne et masculine du cinéma narratif dominant.

Enfin, Flitterman propose une lecture très complexe de l'œuvre d'Agnès Varda, « mère de la Nouvelle vague ", et en particulier de Cléo de 5 à 7 (1963) et Sans toit ni loi (1986). Dans le premier, la cinéaste suit l'itinéraire d'une chanteuse de variétés qui, à la faveur d'une crise personnelle (elle craint d'avoir un cancer), va passer du statut d'objet à celui de sujet du regard. Agnès Varda s'interroge à la fois sur le statut des femmes comme images, et sur la façon dont le cinéma construit ces images. Elle rend visible l'écriture filmique pour amener le spectateur à une distance critique, et prend le contre-pied du cinéma hollywoodien qui réduit les femmes à n'être que des images. Avec Sans toit ni loi, la cinéaste déconstruit les mythes romantiques (masculins) de la liberté et de la femme mystérieuse. À travers le récit des derniers jours d'une jeune femme retrouvée morte dans un fossé, Agnès Varda trace «le portrait impossible » de Mona (Sandrine Bonnaire) dont le manque d'ancrage social est articulé à son absence d'image d'elle-même, et l'amène à l'autodestruction. Selon Flitterman, ce film, en refusant de transformer le personnage et le corps féminin en objet sexuel, prend le contre-pied du cinéma dominant.

Flitterman met en évidence la diversité des regards qui construisent, dans le contexte de leur époque, des femmes cinéastes qui se revendiquent, explicitement ou non, comme féministes. On peut regretter que l'ouvrage n'étudie pas l'œuvre de Jacqueline Audry, qui réalisa seize films entre 1946 et 1967, dans le contexte du cinéma grand public de l'après-guerre, en explorant le désir féminin et la construction sociale de l'identité féminine.

18 Jackie Stacey explore dans Star Gazing. Hollywood Cinema and Female Spectatorship (1994) la mémoire des spectatrices britanniques des années 1940 et 1950 pour comprendre la place et la fonction des stars féminines hollywoodiennes, à partir des magazines populaires de cinéma de l'époque et des lettres reçues en réponse à un questionnaire publié dans deux de ses magazines. Se plaçant dans une perspective résolument historique, tout en utilisant une approche psychanalytique féministe ainsi que les acquis des cultural studies sur l'articulation entre texte et audience dans le processus de production du sens, Stacey analyse les fantasmes de la séduction féminine des femmes britanniques de la classe moyenne (blanche) pendant et après la guerre. Cela passe par une étude de la fréquentation du cinéma en Grande-Bretagne à cette période, et de la place du cinéma hollywoodien et de ses stars en relation avec l'idée d'évasion, et de la dimension sexuée de ce phénomène. Elle étudie les raisons spécifiques qui poussent les femmes à fréquenter les cinémas, et les dimensions de cette pratique culturelle à l'intérieur et à l'extérieur des salles obscures. Elle explore ensuite le lien entre les images de stars sur l'écran et la construction de l'identité des spectatrices à cette époque. 

années 1940 et 1950. Dans quelle mesure les stars incitent-elles les spectatrices à consommer, si bien qu'elle deviennent elles-mêmes de objets de consommation pour autrui ? Elle conclut son étude sur les changements culturels dont témoigne ce rapport des spectatrices aux stars. La nostalgie pour une époque où « les stars étaient vraiment des stars » s'articule au désir d'un temps révolu et met en évidence les changements dans le mode de perception filmique qui commence dans les années 1950.

\section{BIBLIOGRAPHIE}

BOBO Jacqueline, 1995, Black Women as Cultural Readers, New York, Columbia University Press.

BURCH Noël, 1994, Revoir Hollywood, Paris, Nathan.

BUTLER Judith, 1990, Gender Trouble: Feminism and the Subversion of Identity, Londres et New York, Routledge.

DOANE Mary Ann, 1988-1989, « Masquerade reconsidered: Further thoughts on the Female Spectator », Discourse, II.I, pp. 42-54.

-, 1982, «Film and Masquerade: theorizing the female spectator », Screen, 23.3-4, pp. 74-88.

DOTY Alexandre, 1993, Making Things Perfectly: Interpreting Mass Culture, Minneapolis, University of Minnesota Press.

DYER Richard, 1990, Now you see it: Studies on Lesbian and Gay film, Londres et New York, Routledge.

-, 1987, Heavenly bodies: Film stars and society, Londres, Macmillan.

-, 1979, Stars, Londres, British Film Institute.

FISCHER Lucy, 1996, Cinematernity. Film, Motherhood and Genre, Princeton, Princeton University Press.

FLITTERMAN-LEWIS Sandy, 1990, To Desire Differently, Feminsim and the French Cinema, New York, Columbia University Press.

GEVER Martha et al., 1993, Queer looks: Perspectives on Lesbian and Gay Film and Video, Londres et New York, Routledge.

GLEDHILL Christine (dir.), 1987, Home is where the heart is: Studies in Melodrama and the Woman's film, Londres, British Film Institute.

JOHNSTON Claire (dir.), 1975, The Works of Dorothy Azner: Towards a Feminist Criticism, Londres, British Film Institute.

KRUTNICK Frank, 1991, In a lonely street: Film noir, Genre, Masculinity, Londres, Routledge.

KUHN Annette, 1994, Women's pictures: Feminism and Cinema, Londres et New York, Verso.

MAYNE Judith, 1990, The Woman at the keyhole: Feminism and Women's Cinema, Bloomington, Indiana University Press.

Clio. Femmes, Genre, Histoire, 10 | 1999 
MERK Mandy, 1993, Perversions: Deviant readings, Londres, Virago.

MOLDESKI Tania, 1988, The Women who knew too much: Hitchcock and Feminist Theory, Londres et New York, Routledge.

Nouvelles questions féministes, 1996, vol. 17, 1, février.

PLANTÉ Christine, 1989, La Petite Sœur de Balzac, Paris, Seuil.

ROLLET Brigitte, 1998, Coline Serreau, Manchester, Manchester University Press.

ROSS Kristin, 1997, Aller plus vite, laver plus blanc. La culture française au tournant des années soixante, Paris, Abbeville.

ROWE Kathleen, 1995, The Unruly Woman. Gender and the Genres of Laughter, Austin, Texas University Press.

RUSSO Vito, 1981, The Celluloid Closet. Homosexuality in the Closet, New York, Harper and Row.

SMITH Alison, 1998, Agnès Varda, Manchester, Manchester University Press.

STACEY Jackie, 1994, Star Gazing: Hollywwod Cinema and Female Spectatorship, Londres et New York, Routledge.

STUDLAR Gaylyn, 1988, In the Realm of Pleasure: Von Sternberg, Dietricht and the Masochistic Aesthetic, New York, Columbia University Press.

TARR Carrie, 1999, Diane Kurys, Manchester, Manchester University Press.

TASKER Yvonne, 1993, Spectacular Bodies: Gender, Genre and the Action cinema, Londres, Routledge.

TURK Edwards B., 1989, Child of Paradise: Marcel Carné and the Golden Age of French Cinema, Cambridge, Harvard University Press.

VINCENDEAU Ginette (avec REYNAUD Bérénice) dir., 1993a, Vingt ans de théories féministes sur le cinéma, CinémAction, 67.

- 1993b, Jean Gabin. Anatomie d'un mythe, Paris, Nathan Université.

WEISS Andrea, 1992, Vampires and Violets: Lesbians in the Cinema, Londres, Jonathan Cape.

\section{NOTES}

1. Pour une analyse du French feminism aux États-Unis, cf. Nouvelles questions féministes, vol. 17, 1, février 1996.

2. Selon Kristin Ross (1997) pourtant, le structuralisme français réussit après la guerre à "conquérir" les sciences humaines, toutes disciplines confondues, il est vrai dans le cadre institutionnellement marginal de l'Ecole des hautes études en sciences sociales.

3. Malgré le pessimisme apparent de ce constat, le récent colloque (septembre 1998) de la non moins récente AFECCAV (Association française des enseignants et chercheurs en cinéma et audiovisuel), semble prouver que certaines approches de ce type émergent dans le monde universitaire français.

4. Kristin Ross 1997 : 195

5. Cette distinction ne date pas du septième art, et les auteures d'œuvres littéraires, entre autres, ont souvent elles aussi été confrontées à cette "censure" indirecte qui n'ose pas dire son nom mais qui s'exerce malgré tout. Voir Christine Planté 1989.

6. Vingt ans de théories féministes sur le cinéma, CinémAction, 67, 1993. 
7. Women and film, Jump cut, Camera Obscura, Sight and Sound et Screen sont quelques unes des revues publiées en Grande-Bretagne et aux États-Unis où se sont élaborées ces orientations de recherche depuis le début des années 1970.

8. Et dont l'on peut trouver des extraits dans le numéro de CinémAction précédemment cité.

9. Voir par exemple, Stacey 1994 ; Mayne 1990 ; Kuhn 1994.

10. Gledhill (dir.) 1987, Krutnick 1991; Tasker 1993 ; Rowe 1995.

11. Moldeski 1988 ; Studlar 1988 ; Turk 1989.

12. Voir Flitterman-Lewis 1990 ; Johnston (dir.) 1975. La nouvelle collection consacrée aux cinéastes français/e/s lancée par l'éditeur britannique Manchester University Press offre aussi trois ouvrages sur des réalisatrices françaises : Agnès Varda d'Alison Smith, 1998 ; Coline Serreau de Brigitte Rollet, 1998 et Diane Kurys de Carrie Tarr, 1999.

13. Bobo 1995.

14. Russo 1981; Weiss 1992.

15. Merck 1993.

16. Dyer 1990 ; Gever et al. 1993.

17. Voir Butler, 1990 ; Doty 1993.

18. Voir ses ouvrages sur le sujet : 1979 et 1987.

19. Vincendeau $1993 \mathrm{~b}$. 